Letter to the Editors

\title{
The Use of a Multi-ISFET Sensor Fabircated in a Single Substrate
}

\section{BART VAN DER SCHOOT, HANS VOORTHUIJZEN and PIET BERGVELD}

Department of Electrical Engineering, Twente University of Technology, $P O$ Box 217 , 7500 AE Enschede (The Netherlands)

(Recelved January 15, 1987, in revsed form May 7, 1987, accepted June 11, 1987)

\section{Abstract}

The consequences of a constant substrate bias on the operation of ISFETs in a source and drain follower curcuit are briefly discussed The blasing of the substrate becomes necessary as only one substrate contact is avalable on a chip that contains several ISFETs Although the gain of the follower circuits therefore becomes smaller than unity, the transfer is practically linear and the application of the ISFETs is not restricted

\section{Introduction}

For the application of ISFETs, a range of different measuring circuits can be used. The purpose of the measuring circuit is to provide an output signal that is proportional to the $\mathrm{pH}$ of the analyte solution. The linearity of this response is first of all dependent on the sensing mechanism, $\imath e$, the potential developed across the interface between solution and gate insulator as a function of $\mathrm{pH}[1]$

On the other hand, there is the transfer characteristic of the electronic curcuitry that should, in the ideal case, directly reflect chemically-induced changes in the interface potential One possible way of doing this is to keep the voltage of the reference electrode constant and, at a constant drain-tosource voltage, $V_{\mathrm{dg}}$, measure the changes in the drain current, $I_{\mathrm{d}}$, as a function of $\mathrm{pH}[2,3]$ Although this method is simple, the transfer will depend on the biasing of the transistor and be different for each device Furthermore, it is not linear due to non-ideal FET characteristics, and thus an extensive calibration is required

Linearity can be improved by the use of an operational amplifier in a feedback loop that controls the potential of the reference electrode [2] In this way, the drain current and the drain-to-source voltage can be held 
constant so that the bias of the ISFET does not change The limitation of this method is, of course, that it can be used for only one ISFET at a time

Alternatively, source and drain follower curcuits [4] can be used, in which also a constant drain-to-source voltage and a constant drain current are maintained In this case, the source voltage changes linearly with the pH-dependent changes in the threshold voltage, while the reference electrode is grounded Thus, this method is suitable for the parallel operation of several ISFETs in the same solution

Another approach is to use a differential ISFET-MOSFET pair in the input stage of an operational amplifier [5,6] If the ISFET and MOSFET are sufficiently matched, the transfer is linear over a wide range At the same time, a compensation for the temperature and light sensitivity of the transistors is obtained This system also works at a constant voltage on the reference electrode and thus is suitable for multi-sensor applications

The chip that we use for our investigations on chemical sensor-actuator systems $[7,8]$ contains ten ISFETs without corresponding MOSFETs Source and drain follower curcuits are used for its operation The transfer of such a system is unity and perfectly linear over a wide input range, at least when bulk (substrate) and source are connected However, since an N-MOS process is used for the fabrication of the multi-ISFETs, there is only one substrate contact avalable As a consequence, the bulk cannot be connected to the source of each individual ISFET because the source voltages need to be controlled independently for all transistors Therefore we make use of a constant substrate voltage of $-15 \mathrm{~V}$ with respect to ground In the follower curcuits, the source voltage is limited between -13 and $+3 \mathrm{~V}$ with respect to ground so that the $\mathrm{p}-\mathrm{n}$ junction between bulk and source will always be reversely biased The consequences of a constant substrate voltage on the operation of the follower circuits will be discussed below

\section{Theoretical considerations}

As the ISFETs are operated in a constant current and a constant drainto-source voltage mode, the source voltage itself is the output signal of the sensor The bulk-to-source voltage will therefore not be constant and this will affect the threshold voltage of the ISFET and thus also the gain of the follower circuit The change in threshold voltage $\left(V_{t}\right)$ of a MOSFET as a function of the bulk-to-source voltage $\left(V_{b s}\right)$ is depicted in Fig 1 [9] It can be described by the following equation [10]

$V_{\mathrm{t}}=V_{\mathrm{fb}}+2 \phi_{\mathrm{f}}+\alpha\left(2 \phi_{\mathrm{f}}-V_{\mathrm{bs}}\right)^{1 / 2}$

in which $V_{\mathrm{fb}}$ is the flat-band voltage, $\phi_{f}$ the Ferm potential difference between doped and undoped sllicon, $\alpha=\left(2 \epsilon_{\mathrm{s}} q N_{\mathrm{a}}\right)^{1 / 2} / C_{\mathrm{ox}}, \epsilon_{\mathrm{s}}=$ semiconductor permittivity, $N_{\mathrm{a}}=$ acceptor impurity concentration and $C_{\mathrm{ox}}=$ oxide capacitance per unit area 


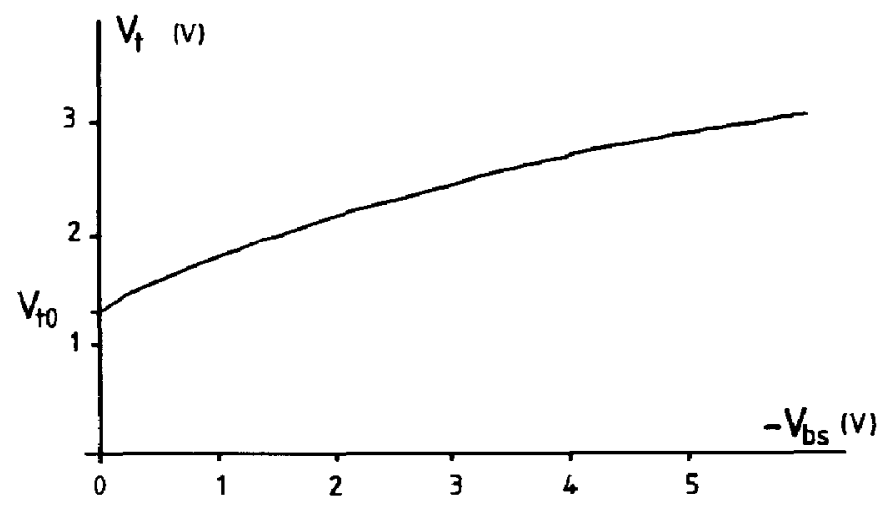

Fig 1 Dependence of $V_{t}$ on bulk-to-source voltage for an n-channel MOSFET (from [9]) $V_{\text {to }}$ indicates the threshold voltage when source and bulk are connected

The flat-band voltage, $V_{\mathrm{fb}}$, of a MOSFET is given by $V_{\mathrm{fb}}=\left(\Phi_{\mathrm{M}}-\Phi_{\mathrm{S}_{1}}\right) / q-\left(Q_{\mathrm{ss}}+Q_{\mathrm{ox}}\right) / C_{\mathrm{ox}}$

$\Phi_{M}$ and $\Phi_{S 1}$ are the work functions of the metal and the sllicon respectively, $q$ is the unit electronic charge and $\left(Q_{\mathrm{gs}}+Q_{\mathrm{ox}}\right) / C_{\mathrm{ox}}$ is the contribution to $V_{\mathrm{t}}$ caused by the charge of the interface states and the charge in the oxide

For an ISFET, the threshold voltage is not only determined by the 'electronic' parameters given in eqns (1) and (2) but also by a chemical term This term can be seen as part of the flat-band voltage [1], which is now given by

$$
V_{\mathrm{fb}}=E_{\mathrm{ref}}-\psi_{\mathrm{o}}+\chi^{\mathrm{sol}}-\Phi_{\mathrm{S}_{1}} / q-\left(Q_{\mathrm{ss}}+Q_{\mathrm{ox}}\right) / C_{\mathrm{ox}}
$$

$E_{\text {ref }}$ is the voltage of the reference electrode including the metal work function $\Phi_{M} / q, \psi_{0}$ is the $\mathrm{pH}$-dependent voltage across the electrolyte-oxide interface and $\chi^{\text {sol }}$ is the surface dipole potential of the solution

In operation, the threshold voltage is affected by two variables, the $\mathrm{pH}$ dependent term $\psi_{0}$ and the bulk-to-source voltage $V_{\mathrm{bs}}$ and thus, for an ISFET, eqns (1) and (3) can be combined and simplified to

$$
V_{\mathrm{t}}=\text { const }-\psi_{\mathrm{o}}+\alpha\left(2 \phi_{\mathrm{f}}-V_{\mathrm{bs}}\right)^{1 / 2}
$$

In first-order MOS theory, the equation for the drain current $\left(I_{\mathrm{d}}\right)$ in the unsaturated region $\left(V_{\mathrm{ds}}<V_{\mathrm{gs}}-V_{\mathrm{t}}\right)$ is

$I_{\mathrm{d}}=\beta\left[\left(V_{\mathrm{gs}}-V_{\mathrm{t}}\right) V_{\mathrm{ds}}-1 / 2 V_{\mathrm{ds}}{ }^{2}\right]$

where $\beta=\mu C_{o x} W / L$, a constant factor in which $\mu$ is the electron mobility and $W / L$ is the width/length ratio of the channel, $V_{\mathrm{gs}}=$ gate-to-source voltage and $V_{\mathrm{ds}}=$ drain-to-source voltage

In the source and drain follower curcuits, $I_{\mathrm{d}}$ and $V_{\mathrm{ds}}$ are kept constant When the reference electrode is grounded, $V_{\mathrm{gs}}$ is equal to $-V_{\mathrm{s}}$, the source voltage with respect to ground Thus eqn. (5) can be rewritten as

$-V_{\mathrm{s}}-V_{\mathrm{t}}=I_{\mathrm{d}} / \beta V_{\mathrm{ds}}+1 / 2 V_{\mathrm{ds}}$ 
$V_{\mathrm{bs}}$ in eqn (4) can also be written as $V_{\mathrm{b}}-V_{\mathrm{s}}$ where $V_{\mathrm{b}}$ is the bulk voltage with respect to ground Combination of eqns (4) and (b) now leads to

$-V_{\mathrm{s}}-\mathrm{const}+\psi_{0}-\alpha\left(2 \phi_{\mathrm{f}}-V_{\mathrm{b}}+V_{\mathrm{s}}\right)^{1 / 2}=I_{\mathrm{d}} / \beta V_{\mathrm{ds}}+1 / 2 V_{\mathrm{ds}}$

This equation can be differentiated to find the relationship between a chemical shift in the threshold voltage and the source voltage, which is the sensor output

$-\delta V_{\mathrm{s}}+\delta\left(\psi_{0}\right)-1 / 2 \alpha\left(2 \phi_{\mathrm{f}}-V_{\mathrm{b}}+V_{\mathrm{s}}\right)^{-1 / 2} \delta V_{\mathrm{s}}=0$

or

$\delta V_{\mathrm{s}}=\left\{1+\alpha /\left[2\left(2 \phi_{\mathrm{f}}-\mathrm{V}_{\mathrm{bs}}\right)^{1 / 2}\right]\right\}^{-1} \delta\left(\psi_{0}\right)$

When values for $\alpha$ and $\phi_{i}$ are substituted into this equation, the gain $\delta V_{\mathrm{s}} / \delta\left(\psi_{\mathrm{o}}\right)$ of the follower circuit as a function of $V_{\mathrm{bs}}$ can be calculated It can be shown that $\alpha$ is approximately $074 \mathrm{~V}^{1 / 2}$ and $\phi_{\mathrm{f}} \approx 03 \mathrm{~V}$ Thus if the substrate is held at a constant voltage, the gain for $V_{b s} \rightarrow 0$ is 068 and increases to 1 for $V_{\mathrm{bs}} \rightarrow-\infty$

\section{Expenmental venfication and conclusion}

Figure 2 shows the gain of the follower as measured for an n-channel MOSFET for different substrate voltages For this experment a MOSFET was used that is completely identical to the ISFETs that we normally apply, with the exception, of course, of a metal gate electrode In this case, $V_{s}$ is given as a function of $V_{g}$ For an ISFET, it is the change in the threshold voltage $V_{t}$ that is the essential sensor signal For a MOSFET, this threshold voltage cannot easlly be modified, but from eqn (5) it follows that $V_{t}$ and $V_{\mathrm{g}}$ are complementary and, thus, $V_{\mathrm{g}}$ is used as the input parameter in this case

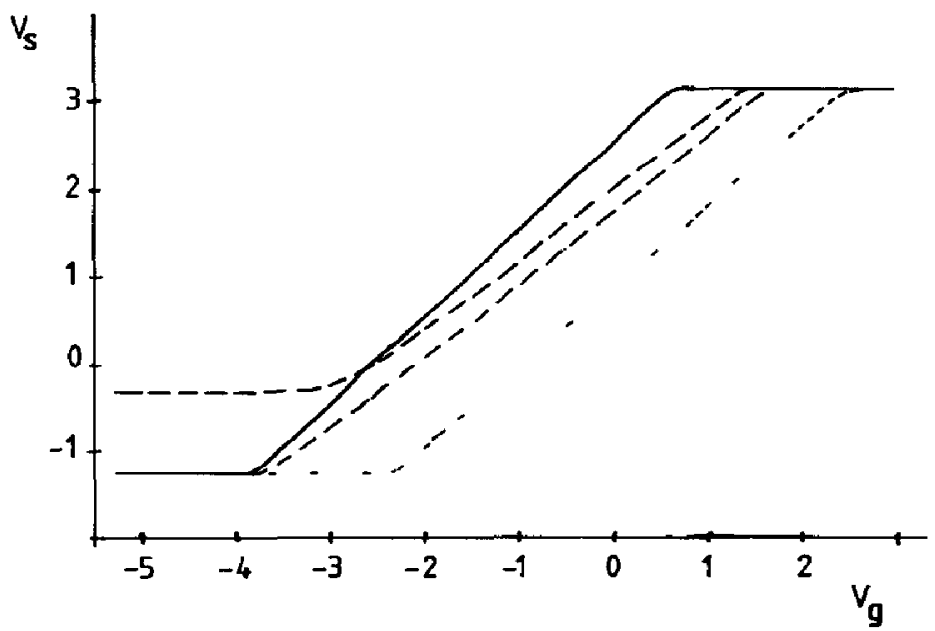

Fig 2 Transfer of the source and drain follower for various values of $V_{b} V_{b}=V_{s}(\longrightarrow)$, $V_{\mathrm{b}}=0 \mathrm{~V}(---), V_{\mathrm{b}}=-15 \mathrm{~V}(--)$ and $V_{\mathrm{b}}=-10 \mathrm{~V}(\cdots,-)$ 
It must be noted that the same experiment can of course be performed by using an ISFET and applying a variable voltage to the reference electrode as the input signal. Of course, the result of this experiment will be exactly identical to that of the MOSFET experiment Alternatively, while using an ISFET, the $\mathrm{pH}$ of the solution can be changed, in which case $V_{t}$ is really modified However, in this way it will be rather difficult to create a continuous registration of the curves as given in Fig. 2 Moreover, by changing the $\mathrm{pH}$ from, for instance, $\mathrm{pH} 2$ to $\mathrm{pH} \mathrm{12,} \mathrm{the} \mathrm{change} \mathrm{in} \mathrm{threshold} \mathrm{voltage}$ is limited to approximately $500 \mathrm{mV}$, depending on the sensitivity of the ISFET It will thus be clear that an 'electrical simulation' of the ISFET is more instructive in this case

If bulk and source are connected, the gain is equal to one From the curve for $V_{\mathrm{b}}=0 \mathrm{~V}$, it can be seen that for values of $V_{\mathrm{s}}<V_{\mathrm{b}}$, the follower cannot operate properly In that case the source-bulk diode is conducting and the current does not flow merely through the FET channel but also through the diode. The curves for $V_{\mathrm{b}}=-1.5 \mathrm{~V}$ and $V_{\mathrm{b}}=-10 \mathrm{~V}$ are also given. It will be clear that the transfer function of the follower becomes more linear as $\left|V_{b s}\right|$ increases For use in connection with ISFETs, however, $V_{s}$ is limited to $-1.5 \mathrm{~V}$ because at large positive potentials of the solution with respect to the substrate, a breakdown of the gate insulator may occur Although $V_{b s}$ is therefore chosen relatively low, the transfer of the follower is practically linear over the working range of the ISFET, because the changes $\mathrm{m} V_{\mathrm{t}}$ as a function of $\mathrm{pH}$ are limited to some hundreds of millivolts

For an ISFET with $V_{\mathrm{t}} \approx 0 \mathrm{~V}, V_{\mathrm{ds}}=500 \mathrm{mV}, I_{\mathrm{d}}=100 \mu \mathrm{A}, V_{\mathrm{b}}=-15 \mathrm{~V}$ and the reference electrode connected to ground, the gain of the follower is found to be approximately 0.8 . This value is in perfect agreement with eqn. (8) The apparent sensitivity of the ISFETs is measured to be $43-45$ $\mathrm{mV} / \mathrm{pH}$ When a correction is applied for the non-unity gain of the followers, this results in a sensitivity of $54-56 \mathrm{mV}$ per decade, which is in agreement with the values found in the literature for $\mathrm{Ta}_{2} \mathrm{O}_{5}$ ISFETs [11]

\section{References}

1 L Bousse, N F de Roolj and P Bergveld, Operation of chemically sensitıve fieldeffect transistors as a function of the insulator-electrolyte interface, IEEE Trans Electron Deurces, ED-30 (1983) 1263 - 1270

$2 \mathrm{~J}$ Janata and $\mathrm{R} J$ Huber, in $H$ Freiser (ed ), Ion Selective Electrodes in A nalytical Chemustry, Vol 2, Plenum Press, New York, 1980, Ch 3

3 A Sibbald, P D Whalley and A K Covngton, A miniature flow-through cell with a four-function ChemFET integrated creut for simultaneous measurements of potassium, hydrogen, calcium and sodium lons, Anal Chim Acta, 159 (1984) $47-62$

4 P Bergveld, Electronic-curcut-design pnnciples for parameter control of ISFETs and related devices, Med Biol Eng, 17 (1979) 655-661

5 C D Fung and C W Fu, The design of on-chip temperature-compensated interface circuits for ISFETs, Proc Transducers' 85', Philadephia, PA, US A, June 11 - 14, 1985 , pp $53-56$ 
6 A Sibbald, A chemical-sensitive integrated circuit, the operational transducer, Sensors and Actuators, 7 (1985) 23 - 38

$7 \mathrm{~B} H$ van der Schoot and $P$ Bergveld, An ISFET-based microliter titrator, integration of a chemical sensor-actuator system, Sensors and Actuators, 8 (1985) 11 - 22

8 B H van der Schoot, Coulometric sensors, Thests, Twente University of Technology, Enschede, The Netherlands, 1986

9 E Vittoz, Design of analog CMOS ICs, Spring Course, Twente University of Technology, 1983

$10 \mathrm{~S}$ M Sze, Physics of Semiconductor Deutces, Wley, New York, 2nd edn, 1981, Ch 8

11 T Akiyama, Y Ujihira, $Y$ Okabe, $T$ Sugano and $E$ Niki, Ion-sensitive field-effect transistors with inorganic gate oxide for $\mathrm{pH}$ sensing, IEEE Trans Electron Devices, ED-29 (1982) $1936-1941$

\section{Blographies}

Bart $H$ van der Schoot received an M S degree in pharmaceutical sciences from Groningen University in the Netherlands in 1982 and a Ph D in technical sciences from Twente University, Enschede, The Netherlands, in 1986

The subject of his Ph D thesis was ISFET-based sensor-actuator systems for prolonged stability in chemical sensing At present, he is working on the use of the sensor-actuator principle in biosensors

He is employed at Twente University as a research fellow for the Royal Netherlands Academy of Arts and Sciences

Johannes Arie Voorthuyzen received an M.S degree in electrical eng1neering from the Delft University of Technology, Delft, The Netherlands, in 1982 In the same year he joined the Bio-information Group, Department of Electrical Engineering, Twente University of Technology, Enschede, The Netherlands, where he received his Ph.D. degree in 1986 His current research is focused on sensors for biomedical applications

Plet Bergueld recelved an M S degree in electrical engineering from Eindhoven University of Technology, Eindhoven, The Netherlands, in 1965, and a Ph.D degree from Twente University, Enschede, the Netherlands, in 1973. In 1984 he was appointed as full professor in biosensor technology at the Twente University of Technology He is chaurman of the Research Unit Sensors and Actuators of this University He is involved in research on electronic measuring and stimulating methods in physiological systems, with special attention for in vivo biosensors He lectures on biomedical instrumentation for graduate students. 